\title{
A Proposal for a Standard Communication Protocol for Pacemaker/ICD Programmers
}

\author{
WA Dijk $^{1,2}$, CAM Hooijschuur ${ }^{1,2}$, W van der Velde ${ }^{1}$, WRM Dassen ${ }^{3,2}$ \\ ${ }^{1}$ University Medical Center Groningen, Groningen, The Netherlands \\ ${ }^{2}$ Dutch Pacemaker Registration Foundation, Groningen, The Netherlands \\ ${ }^{3}$ Maastricht University, Maastricht, The Netherlands
}

\begin{abstract}
The information generated by pacemakers and ICD's to support the cardiologist and technician for installing the optimal settings for the patient is increasing rapidly. In this paper a proposal is described for electronic data exchange between the pacemaker/ICD programmers and electronic information systems.
\end{abstract}

\section{Introduction}

The contemporary pacemakers and implantable cardiac defibrillators (ICD) are complex hybrid devices (1). Their performance can be optimally tuned to the patient's needs based on the cardiac deficiencies he / she is suffering from. Furthermore they are able to store relevant information during the periods in between the visits to the follow-up clinic. This information, ranging from the number of detected premature ventricular complexes to the trend of the sense thresholds, supports the pacemaker technician in choosing the patient related optimal settings of the device.

The communication between the implanted device and the technician is realised through a so called pacemaker programmer. These programmers are different per pacemaker/ICD manufacturer and even not always downward compatible for the different pacemaker models from the same company! Consequently each clinic has a number of programmers in order to be able to monitor all their patients (fig. 1).

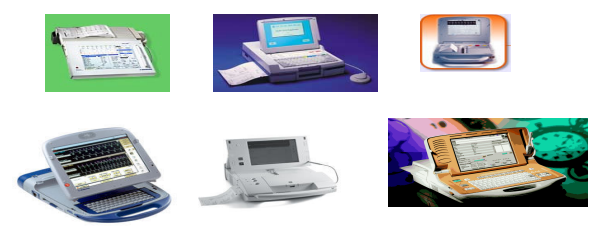

Figure 1. Pacemaker/ICD programmers
This inevitable fact not only causes the technician to serve a variety of user interfaces, but he / she also has to interpret the different data that is generated through the programmers. Figure 2 illustrates the output of 3 different programmers. Corresponding measurements are placed in different positions on the sheet, presented in different units, etc..

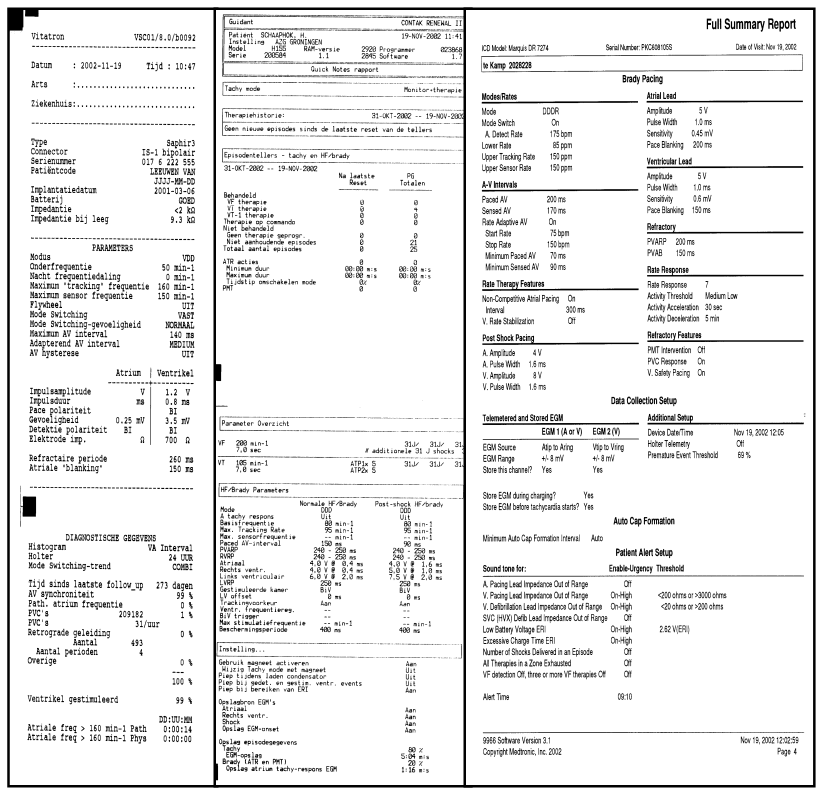

Figure 2. Paper output from 3 different programmers

As electronic patient dossiers are quite common, the above mentioned diverse approaches per programmer have become even more complex due to the administrative burden of interpretation and entering the information into the database in a standard manner.

A direct connection between the programmers and the database, independent from the manufacturer, would decrease the number of interpretation errors and the administrative workload significantly.

We therefor propose a strictly defined communication 
protocol based on HL7, supported by all manufacturers.

\section{Methods}

In 1998 an integrity check was performed on the data collected in the Central Pacemaker Patient Registry (CPPR) where information is stored of all implantations of the 108 pacemaker clinics in the Netherlands since 1976 (2). This investigation revealed 2 major shortcomings:

1. The majority of the errors showed up in the serial numbers of the devices, resulting in a partial fulfilling of the goal to be able to trace malfunctioning pacemakers and ICD's.

2. Pacemaker models lived their own life: many clinics defined their own nomenclature for pacemaker and ICD, giving rise to misinterpretations in the central database.

To minimise the errors introduced by transmitting the data through paper a project was started to realise a well defined electronic communication path between the different clinics and the CPPR, resulting in a minimal leftover workload of typing. At the same time the companies expressed their policies of withdrawal to core business, which is of course manufacturing and/or selling pacemakers and ICD's. Before that change in policy they had introduced and supported a couple of electronic patient dossiers. Maintaining these databases proved to be very time and money consuming. Under guidance of the Dutch Working Group on Cardiac Pacing a surveillance of the existing databases was performed and one was selected to be introduced as being the standard information system for pacemaker patients in the (near) future. This package, called GRIT-SPRN, developed in the University Medical Center in Groningen, the Netherlands (3), written in Visual Foxpro 6.0 (4) underwent major modifications in order to support the above mentioned goals:

1. Information collected on implantations could be transmitted electronically to the CPPR in a standardised way resulting in a one to one correspondence between the data in the clinics and at the central registry. As the electronic infrastructure in each of the participating hospitals varies, a construction was chosen where the (encrypted) information is send as an attachment of electronic mail. Once arrived at the central registry, the data is automatically decrypted and stored.

2. Information on the models is distributed through a web site maintained by the central registry. This information, stored in files, has to be downloaded before being able to enter new models in the local information system.

Next to the considerations mentioned above the copying process of information produced by the pacemaker programmers is another administrative burden and potential error source. The different programmers export their data in a diverse manner, by means of digital files, paper or only displayed on a monitor. Retrieving information from these different sources is prone to reading errors and misinterpretations. We therefor evaluated the possibilities of direct connection between the programmers and the GRIT-SPRN PC.

Tailor made connections between the different programmers and the $\mathrm{PC}$ will result in hard to maintain infrastructures. Standard communication language like the predominantly used Health Level-7 (5) for alphanumeric data exchange bypasses these potential problems because:

1. The data is described in a unique well documented way, leaving no space for misinterpretations.

2. New or modified equipment conforming to the standard can be incorporated with little or no effort.

As no HL-7 data definitions exists for exchanging pacemaker/ICD related information, we made a proposal for such a definition, abiding each applicable requirement demanded by the HL-7 working group.

\section{Results}

A HL-7 message is composed of different segments, each with its specific function and structure. A message starts with a message header (MSH) followed by patient identification (PID), observation requests (OBR) and observation/results (OBX). The MSH segment defines the intent, source, destination and some specifics of the syntax of a message like the character used as field separator. The PID segment contains patient ID and demographic information that is not likely to change. The OBR segment serves as a report header, identifying the observation set represented by the following atomic observations. The actual measurement information, including data types, description and units, is stored in the OBX segments. Each single observation or observation fragment is stored in an OBX segment. The maximum number of possible OBX segments is equal to the number of fields in the database liable to be filled by data from the programmers. In the current GRIT-SPRN database 57 fields are directly related to programmer information. Fig. 3 displays all the different fields of such a HL-7 message.

We started a pilot project in order to investigate the potentials of using HL-7. Giving the facts that the amount of information to be transmitted is relatively small and that all the equipment resides on the same desk, a simple serial connection based on RS232 (or Blue Tooth) fulfils the requirements. 
MSH|^ $|\&|$ Programmer-ID\|GRIT-SPRN||20040419150859||ORU^R01|20040419150856|P|2.4 PIDII0301538\|

OBRI1II^Pacemaker Follow-up|||20040419150857

OBXI1|NM|Atrium Paced $\|92|\%|\||| \mid \mathrm{F}$

OBX|2|NM|Ventricle Paced $\| 87|\%||||| \mid F$

OBXI3|NM|Atrium Sensed $\|8|\%|\||| \mid \mathrm{F}$

OBX|4|NMIVentricle Sensed||13|\% ||||||F

OBXI5|NM|AV Synchrony $\|100|\%|\||| \mid F$

OBXI6|NM|Premature Ventricular Complex Per Hour||0.09|/hr|||||F

OBXI7|NM|P Hist Kl Bin $\| 0|\%||||| \mathrm{F}$

OBXI8INM|Days Since Last Visitl|163||||||F

OBX|9|NMIVentricular Safety Pacel|0.031/hr||l||F

OBXI10|NM|Premature Ventricular Complex\|||347||||||F

OBXI11INM|Atrial Lead Impendance Unill650lohm|l|||F

OBXI12|NM|Atrial Pulse Energy Unill5.5luj|l|||F

OBXI13|NM|Ventricular Lead Impedance Unill500lohm||l||F

OBXI14|NM|Ventricular Pulse Energy Unill5.5luj|l||||F

OBX|15|NM|Battery Impedancell100.035|kohm|l|||F

OBXI16INM|Battery Voltage $|2.80| v|||| \mid F$

OBXI17|NM|Battery Current||"'|ual||||F

OBXI18IST|Battery Status $\|$ good||||||F

OBXI19|NM|Atrial Average Pulse Current Unil|4.5luall|||F

OBX|20|NM|Atrial Average Pulse Current Bill4.5luall|l|F

OBXI21/NMIVentricular Average Pulse Current Unill5.333luall|||F

OBX|22INMIVentricular Average Pulse Current Bill5.333luall|||F

OBX|23INM|Atrial Lead Impedance Bill650lohm|||||F

OBXI24INM|Atrial Pulse Energy Bill5.5luall|l|IF

OBX|25|NMIVentricular Lead Impedance Bill600lohm||l|||F

OBX|26|NM|Ventricular Pulse Energy Bill5.333luj|l|||F

OBXI27|STIModel|VVTIIIIIIF

OBXI28INM|Lower Rate Limit||6|/min|||||F

OBX|29|NM|Upper Rate Tracking $\|140|/ \mathrm{min} \|||| \mid \mathrm{F}$

OBXI30|NMIUpper Rate Sensing $\|44|/ \mathrm{min}\||\|| \mathrm{F}$

OBX|31INMISleepratell60|/min IIIIIF

OBXI32INMIMaximum PAV Delayll|ms $|\|||| F$

OBXI33|NMIMaximum SAV Delay||"'|ms||||||F

OBX|34|ST|Adaptive AV Delay||"'"||||||F

OBXI35|STIAV Delay Hysteresis/lunknown|||||||F

OBX|36|ST|Atrial Sense Polarityllbill||l||F

OBX|37|ST|Atrial Pace Polarityllunil||||||F

OBXI38ISTIVentricular Sense Polarityllunil||||||F

OBX|39|STIVentricular Pace Polarityllbil|ll||F

OBX|40|NM|Ventricular Refractory||260.333|ms||||||F

OBX|41INM|Atrial Blanking $\|150.333|\mathrm{~ms}\||\|| \mathrm{F}$

OBX|42|NMIVentricular Blanking || $30.333|\mathrm{~ms}||||| \mathrm{F}$

OBX|43|NM|Atrial Sensitivity|l0.333|mv||l|||F

OBX|44|NMIVentricular Sensitivity||2.333|mv||||||F

OBX|45|ST|Activity Sensellmedium high|||||||F

OBX|46|NM|Atrial Amplitudel|3.333|v|l|||F

OBX|47|NM|Atrial Pulse Duration || $0.333|\mathrm{~ms}||||| \mathrm{F}$

OBXI48INMIVentricular Amplitudell3.333|v|l||||F

OBX|49|NMIVentricular Pulse Duration $\|0.333|\mathrm{~ms} \|||| \mid \mathrm{F}$

OBX|50|ST|Modeswitch\|fixed |||||| $\mid F$

OBXI51ISTISensor Blending $\|\mathrm{ACT}|\|||| \mathrm{F}$

OBXI52IST|Flywheel|loff||||||F

OBXI53ISTIPVC Synchronous Atrial Stimulation\|lunknown||||||F

OBXI54INM|Atrial Sychronous Pulse Intervall|300.333/ms ||||||$F$

OBX|55|STIVentricular Safety Pacing $\|$ fixed $\||||| \mid F$

OBXI56INM|Hysteresis I|0.3331/min|||||F

OBX|57|NM|Atrial Refractory || $8.333|\mathrm{~ms}||||| \mathrm{F}$

Figure 3. The proposed complete dataset 
The information produced by a programmer of one of the manufacturers, was converted into a file with the standard HL-7 structure and according to the proposed content definition. A PC was used to mimic the programmer. This "programmer" was then connected through the serial communication ports with the GRITSPRN PC. As in Visual Foxpro the addressing of the serial communication ports is cumbersome, a program written in Java was used for this purpose. This program can be launched by Visual Foxpro and is parameter driven, giving it therefor full control. The GRIT-SPRN PC puts itself in a server mode waiting for the "programmer" to act. X-modem is the basic communication protocol between the two machines. The HL-7 message exchange is used for higher layer communication. Upon completion of the communication session, a file containing the desired information is stored in a predefined location and accessible for further processing by the GRIT software.

\section{Discussion and conclusions}

The communication between programmers and information systems based on the proposed HL-7 description proved to be feasible. Although some additional procedures have to be undertaken, the overall functionality is significantly improved:

1. Less copying procedures resulting in less errors

2. Diminished workload

3. Well defined information and communication procedures resulting in enhanced flexibility.

\section{Acknowledgements}

We thank H. Keuning for her help in designing and testing the pilot.

\section{References}

[1] Haddad SAP, Houben R and Serdijn, WA. The history and development of pacemakers: an electronics perspective. Klinische Fysica 8-15. ISSN 0 168-7026.

[2] Dijk WA, Kingma T, Hooijschuur CAM, Dassen WRM, Hoorntje JCA. Validation of the Netherlands Pacemaker Patient Registry. Computers in Cardiology, Lund: 653-656 (1997).

[3] Hooijschuur CAM, Dijk WA, van der Velde W, Sanon B, Ammeraal C, Dassen WRM, Spruijt HJ, Uniform Pacemaker and ICD Information System in The Netherlands. Computers in Cardiology, Memphis: 377-380 (2002).

[4] Stroo E. Microsoft Visual Foxpro 6.0 Language Reference / Microsoft Corporation. ISBN 1-57231-870-8 (1998)

[5] HL7 Standard Version 2.4. An Application Protocol for Electronic Data Exchange in Healthcare Environments. Health Level Seven 3300 Washtenaw, Suite 227 Ann Arbor, MI.

Address for correspondence

W.A. Dijk

University Medical Center

Thorax Center

Hanzeplein 1

9713 EZ Groningen

The Netherlands

W.A.Dijk@Thorax.umcg.nl 